\title{
Hydroxyurea associated ileocecal valve ulcer: evidence for causality
}

\author{
Thevaraajan Jayaraman ${ }^{1}$, Ruveena Bhavani Rajaram², Gin Gin Gan ${ }^{2}$, Ida Hilmi \\ ${ }^{1}$ Gastroenterology Unit, Faculty of Medicine, Universiti Teknologi MARA, Selangor; ${ }^{2}$ Department of Medicine, University of Malaya Medical \\ Centre, Kuala Lumpur, Malaysia
}

Hydroxyurea is an antimetabolite drug that is commonly used in many hematological disorders. Ulcer formation in the gastrointestinal tract is a rare phenomenon associated with this drug. We report a case of a 73-year-old woman who was found to have an isolated ileocecal valve ulcer while on hydroxyurea $1 \mathrm{~g}$ daily for essential thrombocythemia. A comprehensive evaluation ruled out all other causes. The cytoreductive therapy was switched to anagrelide and the endoscopic evaluation 6 months later showed complete healing of the ulcer. However, the hydroxyurea was resumed due to increasing platelet counts and intolerance to dose increments of the anagrelide. Subsequently, the patient was found to have a recurrence of the ulcer. Apart from oral ulcers, there have also been reports of ulcers involving the small bowel and the colon associated with the use of hydroxyurea. The pathophysiology of the non-oral gastrointestinal ulceration in relation to this drug is unclear. Withdrawal of the drug typically leads to complete resolution. Increasing awareness of the rare association between the use of hydroxyurea and nonoral gastrointestinal ulcers is essential for early detection to prevent related complications. (Intest Res 2021;19:468-471)

Key Words: Hydroxyurea; Ulcer; Gastrointestinal tract; Essential thrombocythemia

\section{INTRODUCTION}

Hydroxyurea is an antimetabolite agent commonly used to treat a variety of hematological conditions and was discovered in 1869 by Dresler and Stein. ${ }^{1}$ Hydroxyurea, although generally well tolerated, can cause a wide range of side effects including leukopenia, stomatitis, myelosuppression, mucositis, diarrhea, fever, hepatitis, hyperbilirubinemia, and mucocutaneous changes such hyperpigmentation of the skin and nails, alopecia, dermatomyositis-like eruptions and oral ulcers. ${ }^{2}$ Non-oral gastrointestinal tract ulcer associated with hydroxyurea is a very rare phenomena and herein we report our experience in managing one of such cases. Written informed consent was obtained.

Received September 16, 2020. Revised October 18, 2020.

Accepted October 29, 2020.

Correspondence to Thevaraajan Jayaraman, Gastroenterology Unit, Faculty

of Medicine, Universiti Teknologi MARA, Jalan Hospital, 47000 Sungai Buloh,

Selangor, Malaysia. Tel: +60-3-61265000, Fax: +60-3-61265224, E-mail:

theva.jayaraman@gmail.com

\section{CASE REPORT}

A 73-year-old woman was referred for investigation of unexplained loss of appetite and loss of weight. She had a past medical history of essential thrombocythemia which was positive for JAK2 V617F mutation and was treated with hydroxyurea 1 $\mathrm{g}$ daily for 10 years. She was not on any other long-term medication. Her clinical examination was unremarkable apart from the presence of melanonychia. Her full blood count results were as following; hemoglobin of $11.6 \mathrm{~g} / \mathrm{dL}$, white cell counts of $5.1 \times 10^{9} / \mathrm{L}$ and platelet counts of $335 \times 10^{9} / \mathrm{L}$. Her biochemical profile, coagulation profile, and serum inflammatory markers were all within normal limits. In view of her symptoms and advanced age, a colonoscopy was performed.

The colonoscopy showed a clean based ulcer measuring 2.5 $\mathrm{cm}$ on the ileocecal valve (ICV), with normal mucosa in the terminal ileum and colon (Fig. 1). Histopathological assessment of the biopsies taken from the ulcer edge was consistent with active colitis and ulcerations with no diagnostic features 


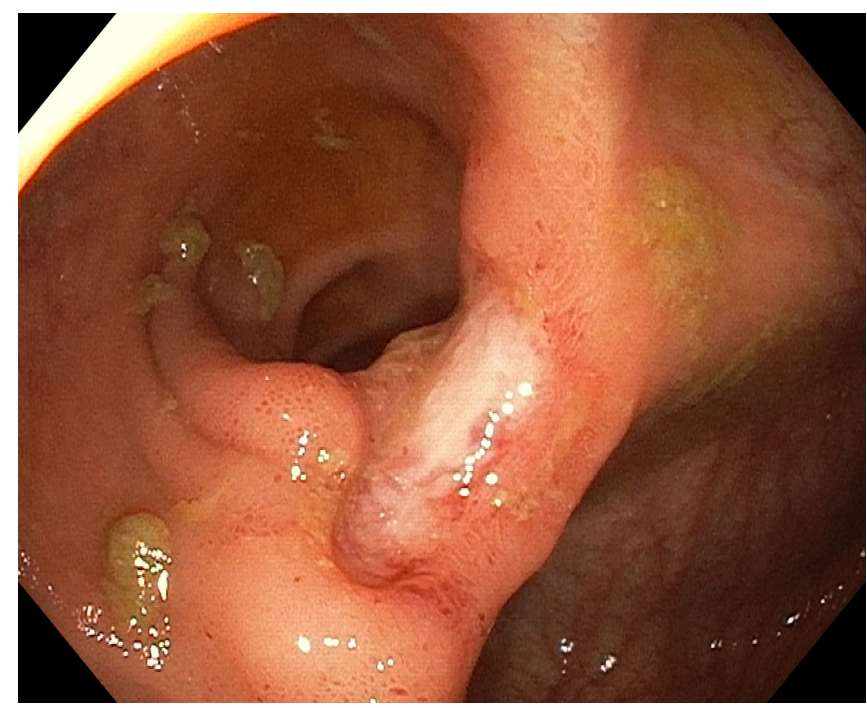

Fig. 1. Isolated ulcer involving the ileocecal valve found on first colonoscopy.

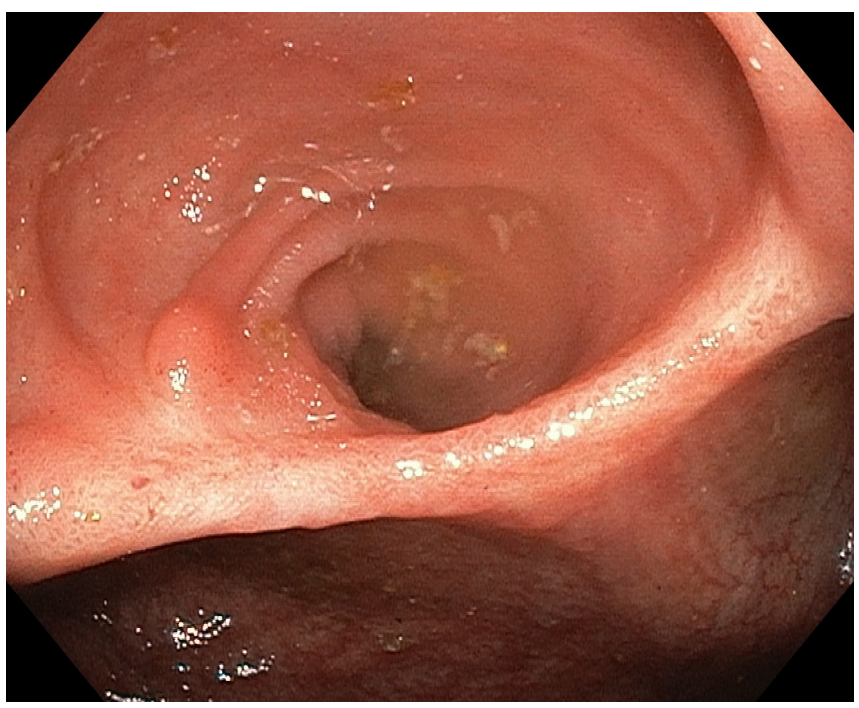

Fig. 2. Complete healing of the ileocecal valve ulcer following cessation of the hydroxyurea as seen during the second colonoscopy.

of tuberculosis (TB) or other pathologies. A serum interferon gamma release assay for TB (T-SPOT ${ }^{\circledR}$.TB; Oxford Immunotec, Ltd., Abingdon, UK) was negative. A computed tomography scan of the thorax, abdomen and pelvis was performed which also yielded no evidence of TB or malignancy. Following this, the solitary ICV ulcer was attributed to the hydroxyurea therapy and patient was counseled to switch to an alternative cytoreductive agent considering the possibility of the ulcer progressing and developing related complications.

The patient agreed to stop the hydroxyurea and was com-

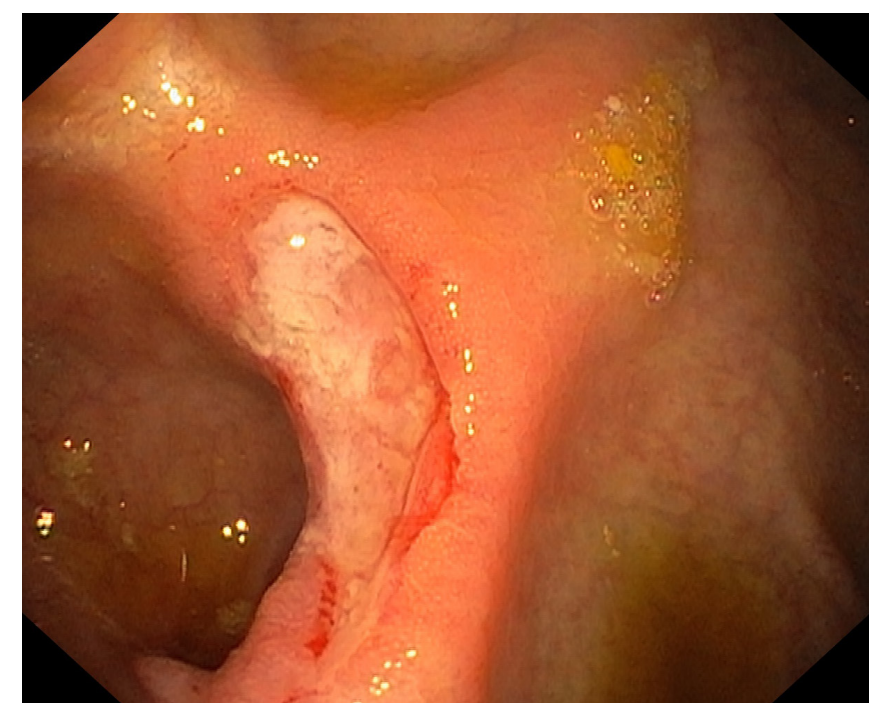

Fig. 3. Recurrence of the ileocecal valve ulcer following resumption of the hydroxyurea as seen during the third colonoscopy.

menced on anagrelide 1,000 $\mu$ g daily in divided doses 3 months after the index colonoscopy. Six months after the index colonoscopy and 4 months after stopping the hydroxyurea, a second colonoscopy was done which showed that the previously seen ICV ulcer has completely healed (Fig. 2). However, 5 months since stopping the hydroxyurea, patient's platelet counts were steadily rising with a zenith of $1,098 \times 10^{9} / \mathrm{L}$ despite incremental dose adjustments of anagrelide to $1,500 \mu \mathrm{g}$ daily in divided doses. Due to the increasing platelet counts the option of reintroduction of the hydroxyurea was discussed with the patient, taking into the account the risk of possible colonic ulcer recurrence. Patient agreed to this option and hydroxyurea was reintroduced at $500 \mathrm{mg}$ on alternate days in addition to the current dose of anagrelide.

Subsequently the platelet counts remained elevated and dose escalation of anagrelide was not possible as the patient started experiencing headache, visual disturbance and dizziness which resolved upon stopping the drug. Patient was counseled for change of therapy to interferon or ruxolitinib. However, patient was not keen on both drugs due to concerns regarding side effects and expensive cost. Patient expressed preference to restart the hydroxyurea and the dose was gradually escalated to $2 \mathrm{~g}$ daily over the course of 6 months following which the platelet counts improved to $425 \times 10^{9} / \mathrm{L}$. Patient then started complaining of loss of appetite and developed an aphthous oral ulcer. A third colonoscopy was done which showed a recurrence of the ICV ulcer (Fig. 3); histopathological assessment of the biopsy specimen from the ulcer edge was negative 
Table 1. Comparison of Reported Cases of Non-oral Gastrointestinal Tract Ulcers Associated with Hydroxyurea Use

\begin{tabular}{lcllllll}
\hline Case No. & Age (yr) & Ethnicity & Sex & Hematological diagnosis & HU dose/duration & Ulcer site & Ulcer pathophysiology \\
\hline $1^{14}$ & 64 & Caucasian & M & Myelofibrosis & NA & Jejunum & Hypersensitivity vasculitis \\
$2^{15}$ & 77 & Japanese & M & Essential thrombocythemia & NA & Duo \& colon & Intestinal Behçet disease \\
$3^{16}$ & 37 & Thai & M & Hemoglobin E-beta-thalassemia & $4.5 \mathrm{~g}$ weekly/3 yr & TI \& colon & Nonspecific inflammation \\
$4^{a}$ & 73 & Chinese & F & Essential thrombocythemia & $7 \mathrm{~g}$ weekly/10 yr & ICV & Nonspecific inflammation \\
\hline
\end{tabular}

${ }^{\text {a }}$ Patient presented in this case report.

HU, hydroxyurea; M, male; F, female; NA, not available; Duo, duodenum; TI, terminal ileum; ICV, ileocecal valve.

for TB and other pathologies. In view of this, patient was advised to stop the hydroxyurea indefinitely and consider another cytoreductive therapy. The patient has since agreed to consider starting interferon therapy.

\section{DISCUSSION}

Hydroxyurea blocks DNA synthesis through inhibition of the ribonucleotide reductase enzyme and is commonly used in the hematological setting to treat myeloproliferative conditions such polycythemia rubra vera, essential thrombocythemia and primary myelofibrosis. ${ }^{1,2}$ There are numerous side effects related to its use including severe mucocutaneous changes such as ulcers involving the acral areas of the leg, genitalia and oral cavity. ${ }^{3}$ The rate of intolerance to hydroxyurea due to side effects has been reported to be between $5 \%$ and $16 \%{ }^{4-9}$

The oral cavity is by far the commonest site for ulcer formation in the gastrointestinal tract associated with hydroxyurea, ${ }^{10-13}$ and oral ulcers were reported to occur in $0.8 \%$ of patients with myeloproliferative neoplasm on hydroxyurea therapy. ${ }^{8}$ Ulcers in the other parts of the gastrointestinal tract are exceedingly rare and to our knowledge, there have been only 3 such reported cases as summarized in Table 1. Of note, cases 1 and 3 underwent emergency surgery for severe gastrointestinal bleed resulting in a wedge resection of the jejunal ulcer and partial right hemicolectomy respectively. All 3 cases had improvement and/or no recurrence after stopping the hydroxyurea therapy; lending credence to the suggested causation between the gastrointestinal ulcers and exposure to hydroxyurea. ${ }^{14-16}$

The pathophysiology of gastrointestinal ulcer formation in relation to hydroxyurea therapy is not well understood with drug-induced hypersensitivity vasculitis and intestinal Behçet disease being possible etiologies. ${ }^{14,15}$ In our case, the causal relationship between the drug exposure and ulcer formation has been established based on the resolution of the ulcer upon withdrawal of the offending drug and recurrence of the ulcer upon resumption of the drug in the absence of other potential causes. Although the clinical sequelae of the ulcer formation in our patient was not as severe as the other reported cases, the development of potentially life-threatening complications in the future cannot be ruled out due to our lack of understanding of the pathophysiology and determinants of ulcer progression in relation to hydroxyurea use. Thus, switching the patient to an alternative cytoreductive therapy remains to be the correct decision. Due to the paucity of data on hydroxyurea associated non-oral gastrointestinal ulcer, the effect of dose and duration on ulcer formation is currently unknown.

To determine the true prevalence of non-oral gastrointestinal ulcer in patients receiving hydroxyurea, a screening upper and lower gastrointestinal endoscopy in addition to a small bowel study will be necessary in all patients on hydroxyurea therapy; however, this will have an unfavorable cost-benefit analysis due to the rarity of the ulcers and exorbitant cost associated with the complete examination of the gastrointestinal tract lumen. As such, having a high index of suspicion and judicious testing of symptomatic patients on hydroxyurea therapy is the preferred approach currently. It is hoped that our case report will contribute towards increasing awareness of the very rare but important ulcerogenic potential of hydroxyurea on the non-oral portion of the gastrointestinal tract.

Hydroxyurea can rarely cause non-oral gastrointestinal ulcers and our report represents only 1 of 4 such cases in the published literature to date. A high index of suspicion is needed to investigate and diagnose this rare adverse event which typically resolves upon stopping the drug. Early detection could help prevent potentially life-threatening complications typically associated with the ulcer formations.

\section{ADDITIONAL INFORMATION}

\section{Funding Source}

The authors received no financial support for the research, 
authorship, and/or publication of this article.

\section{Conflict of Interest}

No potential conflict of interest relevant to this article was reported.

\section{Author Contribution}

Conceptualization: Jayaraman T, Hilmi I. Supervision: Gan GG, Hilmi I. Writing - original draft: Jayaraman T, Rajaram RB. Writing - review \& editing: Gan GG, Hilmi I. Approval of final manuscript: all authors.

\section{ORCID}

Jayaraman T https://orcid.org/0000-0003-2035-6410

Rajaram RB https://orcid.org/0000-0002-3855-6536

Gan GG https://orcid.org/0000-0003-2129-782X

Hilmi I https://orcid.org/0000-0001-7091-0032

\section{REFERENCES}

1. Saban N, Bujak M. Hydroxyurea and hydroxamic acid derivatives as antitumor drugs. Cancer Chemother Pharmacol 2009; 64:213-221.

2. Madaan K, Kaushik D, Verma T. Hydroxyurea: a key player in cancer chemotherapy. Expert Rev Anticancer Ther 2012;12: 19-29.

3. Vassallo C, Passamonti F, Merante S, et al. Muco-cutaneous changes during long-term therapy with hydroxyurea in chronic myeloid leukaemia. Clin Exp Dermatol 2001;26:141-148.

4. Randi ML, Ruzzon E, Luzzatto G, Tezza F, Girolami A, Fabris F. Safety profile of hydroxyurea in the treatment of patients with Philadelphia-negative chronic myeloproliferative disorders. Haematologica 2005;90:261-262.

5. Martínez-Trillos A, Gaya A, Maffioli M, et al. Efficacy and tolerability of hydroxyurea in the treatment of the hyperproliferative manifestations of myelofibrosis: results in 40 patients. Ann Hematol 2010;89:1233-1237.

6. Hernández-Boluda JC, Alvarez-Larrán A, Gómez M, et al. Clini- cal evaluation of the European LeukaemiaNet criteria for clinicohaematological response and resistance/intolerance to hydroxycarbamide in essential thrombocythaemia. Br J Haematol 2011;152:81-88.

7. Alvarez-Larrán A, Pereira A, Cervantes F, et al. Assessment and prognostic value of the European LeukemiaNet criteria for clinicohematologic response, resistance, and intolerance to hydroxyurea in polycythemia vera. Blood 2012;119:13631369.

8. Antonioli E, Guglielmelli P, Pieri L, et al. Hydroxyurea-related toxicity in 3,411 patients with Ph'-negative MPN. Am J Hematol 2012;87:552-554.

9. Demuynck T, Verhoef G, Delforge M, Vandenberghe P, Devos T. Polycythemia vera and hydroxyurea resistance/intolerance: a monocentric retrospective analysis. Ann Hematol 2019;98: 1421-1426.

10. Norhaya MR, Cheong SK, Ainoon O, Hamidah NH. Painful oral ulcers with hydroxyurea therapy. Singapore Med J 1997; 38:283-284

11. Paleri V, Lindsey L. Oral ulcers caused by hydroxyurea. J Laryngol Otol 2000;114:976-977.

12. Mendonça R, Gueiros LA, Capellaro K, Pinheiro VR, Lopes MA. Oral lesions associated with hydroxyurea treatment. Indian J Dent Res 2011;22:869-870.

13. Badawi M, Almazrooa S, Azher F, Alsayes F. Hydroxyurea-induced oral ulceration. Oral Surg Oral Med Oral Pathol Oral Radiol 2015;120:e232-e234.

14. Yousuf K, Kathula S, Hillman N, et al. A case of bleeding jejunal ulcer due to vasculitis from hydroxyurea. Am J Gastroenterol 2000;95:2616.

15. Kobune M, Kato J, Kuribayashi K, et al. Essential thrombocythemia associated with incomplete type intestinal Behçet disease during hydroxyurea treatment. Rinsho Ketsueki 2005;46: 1136-1140.

16. Boonyawat K, Wongwaisayawan S, Nitiyanant P, Atichartakarn V. Hydroxyurea and colonic ulcers: a case report. BMC Gastroenterol 2014;14:134. 